\title{
THE RESPONSE OF STRAIN AND PENDULUM SEISMOGRAPHS TO SURFACE WAVES*
}

\author{
By Hugo Benioff and B. Gutenberg
}

\begin{abstract}
The theory of the response of strain and vectorial seismographs to surface waves is reviewed and extended. Preliminary observations are discussed.

\section{INTRODUCTION}

IN A PAPER published some years ago, ${ }^{1}$ one of us showed that the response of the strain seismograph differs considerably from that of the pendulum seismograph and that in consequence a comparison of seismograms written with the two instruments should provide information on wave characteristics which cannot be derived from observations with either instrument alone. Recently, a vectorial pendulum seismograph has been completed at the Seismological Laboratory which also provides information which cannot readily be derived from single-instrument seismographs. ${ }^{2}$ It seemed desirable, therefore, to determine to what extent these newer instruments contribute to the study of seismic waves.
\end{abstract}

\section{Theoretical Response of Strain and Pendulum Seismographs to Surface Waves}

The angular response of a linear strain seismometer to Rayleigh waves varies as $\cos ^{2} \alpha$, where $\alpha$ is the angle between the indicator rod and the direction of propagation of the wave. If the outputs of two mutually perpendicular linear strain instruments are added together, the combined output is proportional to $\left[\cos ^{2} \alpha+\cos ^{2}\right.$ $\left.\left(\alpha+90^{\circ}\right)\right]=1$; in other words, the combined angular response characteristic of two mutually perpendicular strain seismographs to Rayleigh waves is independent of the direction of the incoming wave. ${ }^{3}$ Moreover, this combination has zero response for Love waves, ${ }^{4}$ since the response of a single strain seismograph to Love waves varies as $\sin \alpha \cos \alpha=1 / 2 \sin 2 \alpha$ and the combined outputs of two such instruments oriented $90^{\circ}$ with respect to each other is proportional to $\sin 2 \alpha-\sin 2 \alpha=0$.

For an electromagnetic pendulum seismograph with a period short in comparison with that of the recorded wave, the output electromotive force is proportional to $d^{3} r / d t^{3}$ ( $r=$ ground displacement), whereas in the electromagnetic strain seismograph the output electromotive force is proportional to $d^{2} r / d t^{2}$. In response to sinusoidal ground waves of identical phase the two galvanometer traces will therefore have a phase difference of $90^{\circ}$ when the galvanometers are identical with respect to

* Manuscript received for publication July 23, 1951. The research reported in this document has been made possible through support and sponsorship extended by the Geophysical Research Directorate of the Cambridge Field Station, AMC, U. S. Air Force, under Contract No. AF 19(122) 436. It is published for technical information only and does not represent recommendations or conclusions of the sponsoring agency.

1 Hugo Benioff, “A Linear Strain Seismograph,” Bull. Seism. Soc. Am., 25: 283-309 (1935).

${ }^{2}$ Hugo Benioff, Beno Gutenberg, and C. F. Richter, "Progress Report, Seismological Laboratory, California Institute of Technology, 1950," Trans. Am. Geophys. Union, 32: 749-754 (1951).

${ }^{3}$ Hugo Benioff, "Directional Characteristics of Strain Seismograph Pairs," Bull. Geol. Soc. Am., 60: 1953 (abstract).

4 Ibid. 
period and damping. Since in Rayleigh waves the phases of the horizontal ground displacements and of the vertical ground displacements differ by $90^{\circ}$, it follows that the traces written by an electromagnetic vertical pendulum seismograph and an electromagnetic strain seismograph are in phase with each other when the circuit connections are such that ground impulses upward and compressions are recorded in the same direction on the seismograms. Moreover, since a compression (or dilatation) is a compression (or dilatation) for any direction of wave propagation, it follows that traces of Rayleigh waves written by two strain seismographs of arbitrary relative orientations having galvanometers of identical period and damping characteristics are always in phase with each other (assuming proper circuit connections) even though the amplitudes may differ. The amplitudes of a single linear strain instrument depend upon azimuth and, moreover, its frequency-response characteristic differs from that of the pendulum seismograph. Consequently, in general, the wave shapes of the vertical electromagnetic pendulum and electromagnetic strain seismograph traces are not identical even though the phases are equal. Since records of surface waves written with two combined mutually perpendicular strain seismographs have zero response to Love waves and are independent of wave azimuth for Rayleigh waves, they should correspond closely with records written by a vertical electromagnetic seismograph, since the response of the latter is also independent of azimuth and is zero for Love waves.

These relationships can be used to identify Rayleigh waves by comparison of phases in seismograms written by a vertical-component electromagnetic pendulum seismograph and of a single electromagnetic strain seismograph having proper constants or by two mutually perpendicular identical strain seismographs. Since trace directions of the horizontal component of the Rayleigh-wave ground movements vary with azimuth, whereas the vertical and strain components of these movements do not change with azimuth, comparisons of the directions of the traces written by two horizontal pendulums with those written by a vertical pendulum or with one strain seismograph provide the means for determining the quadrant from which Rayleigh waves arrive. Although in Rayleigh waves the phase relationships are not dependent upon the direction of propagation, the trace directions as written by horizontal pendulums do depend upon azimuth.

For a Love wave of arbitrary ray azimuth, two mutually perpendicular identical strain seismographs have identical responses equal in amplitude and opposite in sign. This follows from the angular response characteristic of the strain seismograph to Love waves which is $1 / 2 \sin 2 \alpha$, where $\alpha$ is the angle between the ray direction and the strain rod. Thus, when $\alpha$ is increased or decreased by $\pi / 2$, this function changes sign but not its value. Consequently, Love waves and SH waves can be identified at sight on strain seismograms of two mutually perpendicular instruments. Moreover, by comparing strain traces with those of two mutually perpendicular horizontal pendulum seismographs, it is possible to determine the quadrant from which Love waves arrive. This results from the relation of the pendulum angular response characteristics, which for $\mathrm{N}-\mathrm{S}$ and $\mathrm{E}-\mathrm{W}$ orientation are sin $\psi$ and sin $(\psi+\pi / 2)=-\cos \psi$ respectively, to the strain angular characteristics which are $1 / 2 \sin 2 \psi$ and $1 / 2 \sin 2(\psi+\pi / 2)=-1 / 2 \sin 2 \psi ; \psi$ is the ray azimuth. The signs of $\sin \psi,-\cos \psi$ and $1 / 2 \sin 2 \psi$ (or $-1 / 2 \sin 2 \psi$ ) exhibit a different order for each quad- 
rant. ${ }^{5}$ For strain and pendulum instruments with identical frequency characteristics (such as mechanical pendulums having periods and damping constants identical with those of the strain galvanometers) the signs of the angular response functions for Love waves having ray azimuths $\psi$ are given in table 1 . It should be noted that in trace comparisons between electromagnetic pendulum seismographs and electromagnetic strain seismographs when all galvanometers have identical periods and damping constants and when the pendulum periods are short compared with the wave periods, the pendulum trace phases lag the strain phases shown in table 1 by $90^{\circ}$.

TABLE 1

Signs of Angular Response Functions for Love Waves Having Ray AzIMUTHS $\psi$

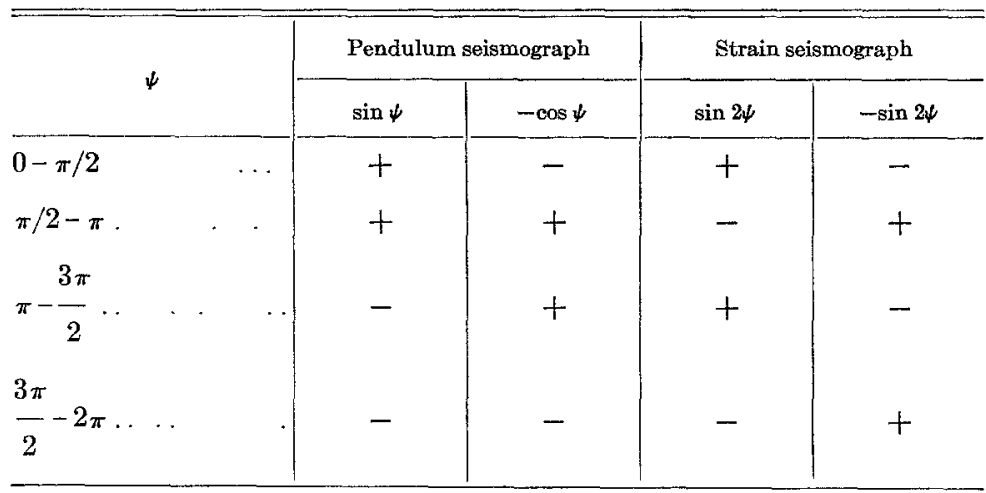

Another useful relationship between pendulum and strain seismograph characteristics was given in an earlier paper. ${ }^{6}$ It was shown that the frequency characteristic of the electromagnetic strain seismograph is identical with that of a simple pendulum seismograph with mechanical or optical recording, if the period and damping coefficients of the strain galvanometer are identical with the period and damping coefficients of the pendulum, or if both are very short or very long in comparison with the wave periods. The traces written with these two instruments are either in phase with each other or differ by $180^{\circ}$, depending upon the direction of arrival of the waves. Therefore, comparisons of traces written by pendulum and strain instruments eliminate the $180^{\circ}$ ambiguity in azimuth which is encountered with observations made with two horizontal pendulums only.

The theoretical considerations given in that paper refer to traveling waves only. They cannot be applied to standing waves.

Since the response of a combined mutually perpendicular strain pair or of a volume strain seismograph is independent of the ray azimuth, it follows that in a vectorial recorder in which one component is provided by a vertical electromagnetic pendulum galvanometer and the other by a combined strain galvanometer the vector response to pure Rayleigh waves is a straight line whose slope is determined by the

5 The application of these signs requires a consistent scheme for designating trace directions and particle displacements.

${ }^{6}$ Benioff, "A Linear Strain Seismograph."

7 Ibid., p. 292. 
ratio of the major and minor axes of the ground-displacement ellipse, assuming equal magnification of the two components. For Love waves, the vertical component is always zero. In a vectorial recorder having a single strain instrument for one component and a vertical pendulum for the other, the vectorial response for Rayleigh waves is a straight line, but the slope of the line is not given by the Rayleigh wave ellipse, since the response of the strain component varies with azimuth.

\section{Observations of Surface Wayes}

With the instrumental installations now available at Pasadena it has been possible to apply some of the principles outlined above to the study of a number of earthquakes which have occurred during the past year. It was found that in teleseisms with transpacific wave paths the surface-wave groups begin as Love waves of several
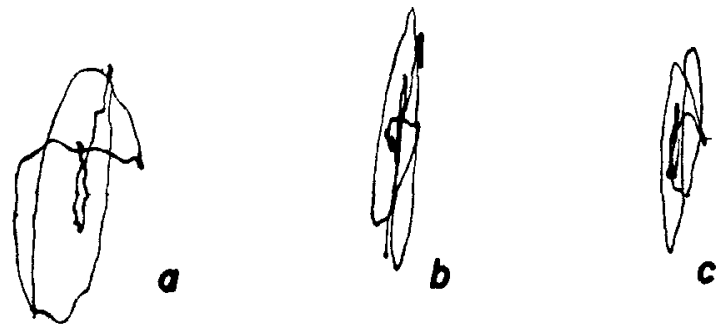

\section{VERTICAL PENDULUM}

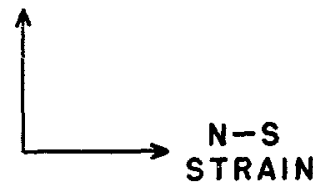

Fig. 1. Pasadena vectorial seismograms, earthquake of 1951 June $6,16^{\text {h }} 10^{\text {mg }}$ G.C.T., near $\phi=71^{\circ} \mathrm{N}, \lambda=8^{\circ} \mathrm{W}$, azimuth approximately $\mathrm{N} 30^{\circ} \mathrm{E}$ : $a$, approximately $16^{\mathrm{h}} 57^{\mathrm{m}} ; b, 17^{\mathrm{h}} 01^{\mathrm{m}} ;$ $c, 17^{\mathrm{h}} 02^{\mathrm{m}}$. Each exposure 30 seconds duration. $T_{g}=10 \mathrm{sec} . \pm$

minutes' duration. ${ }^{8}$ The periods decrease with time. They are followed by Rayleigh waves frequently persisting for more than one hour. The Rayleigh wave trains begin with the longest periods and continue with shorter periods which decrease asymptotically with time. This confirms the coupling theory of Ewing and Press ${ }^{9}$ with respect to the mechanics of Rayleigh-wave propagation under the ocean. In general, microseisms and Love waves are superposed on the Rayleigh waves, but their effects are usually small. The observed directions of motions of the ground particles in Rayleigh waves agree approximately with the direction of the epicenter. Waves propagated over transcontinental paths exhibit irregular changes in amplitude and type. This is illustrated ${ }^{10}$ in figure 1 , which shows sample records from a preliminary vectorial recorder composed of a single strain component and a vertical-pendulum component of unlike magnification. In figure $1, a$, it is apparent that Love waves are superposed on Rayleigh waves. In $b$ and $c$ the recording indicates very nearly pure Rayleigh waves (with superposed microseisms). Horizontal-pendulum vectorial seismograms exhibiting oceanic and continental wave paths have been published in an earlier paper. ${ }^{11}$

In figure 2 selected exposures are reproduced from the horizontal-pendulum vectorial seismograph taken at Pasadena during the passage of surface-wave groups.

${ }^{8}$ B. Gutenberg, "Surface Waves Recorded by a Benioff Vectorial Seismograph," paper presented at the annual meeting of the Seismological Society of America, March 23, 1951.

9 Maurice Ewing and Frank Press, "Surface Waves from Solomon Islands Earthquake, 24 July 1950 " paper presented at the annual meeting named in the preceding footnote.

10 The figures were arranged by $J$. M. Nordquist and L. Paluba.

11 Benioff, Gutenberg, and Richter (as cited in fn. 2 , above), figs. 4 and 5 respectively. 
The arrow indicates the direction of the epicenter. Although some of the wave groups indicate ground vibration parallel to the direction of the epicenter, others depart appreciably from this direction. It should be pointed out that this vectorial seismograph $\left(T_{g}=10 \mathrm{sec}\right.$.) was designed primarily for the recording of microseisms and consequently has much higher magnification for these movements than for the longer-period surface waves. Presumably, most of the short-period irregularities can therefore be attributed to microseismic movements. In figure 3 are shown portions
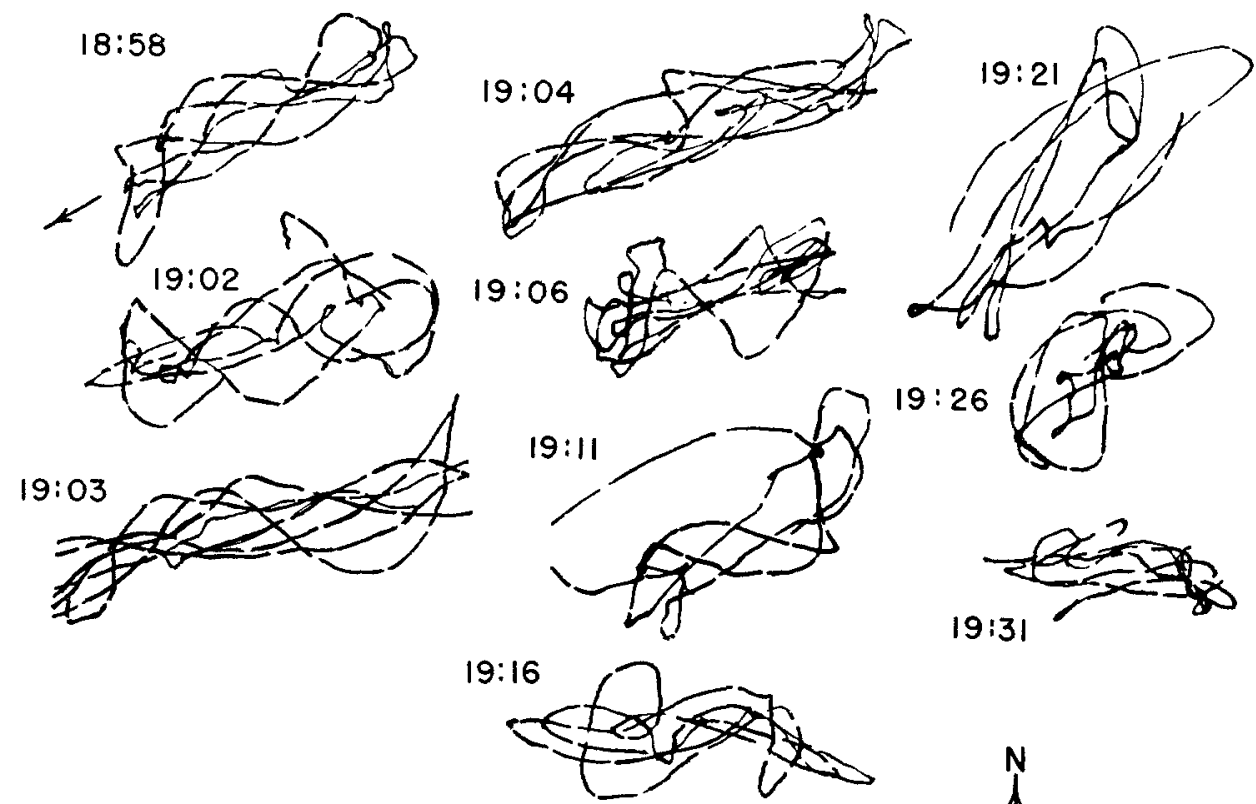

1950 MAY 17

$18: 13.2$

$20^{\circ} \mathrm{S} \quad 169^{\circ} \mathrm{E}$

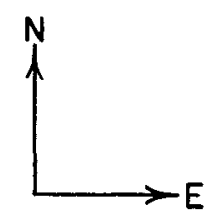

Fig. 2. Pasadena vectorial seismograms. Exposures approximately 50 seconds each. $T_{g}=10$ seconds. Breaks in recording line are at 1-second intervals.

of corresponding seismograms written with ordinary recorders having galvanometer periods of approximately 90 seconds. These include $\mathrm{N}-\mathrm{S}, \mathrm{E}-\mathrm{W}$, and vertical-component electromagnetic pendulum seismographs and $\mathrm{N}-\mathrm{S}$ and $\mathrm{E}-\mathrm{W}$ component electromagnetic linear strain seismographs. In this and subsequent figures the magnification of the $\mathrm{N}-\mathrm{S}$ pendulum is less than that of the $\mathrm{E}-\mathrm{W}$ pendulum, and that of the $\mathrm{E}-\mathrm{W}$ strain is less than the magnification of the $\mathrm{N}-\mathrm{S}$ strain. Moreover, the frequency-response characteristics of the pendulum instruments decrease much more rapidly with period than those of the strain seismographs. In all figures, trace motions toward the top of the seismograms correspond with impulse ground motion up, north, east, and in compression, respectively. In the reproduced portions of the seismograms in figure 3 , the traces of the vertical pendulum and of the two strain instruments are in phase, whereas the traces of the two horizontal pendulums while in phase with each other are displaced relative to those of the strain and vertical 
instruments. This relationship, in addition to the fact that in the corresponding vectorial record (first record in fig. 2) the direction of motion is parallel to the direction of propagation, shows that this group of waves is principally of Rayleigh type. Moreover, the maximum upward trace movements of the two horizontal-pendulum instruments precede the upward trace movements of the other instruments by approximately $90^{\circ}$, corresponding with the predicted characteristic of Rayleigh waves arriving from the southwest (with the recording polarities mentioned earlier).

In figure 4 , parts $a$ and $b$ represent portions of seismograms of a Samoan earthquake which occurred at approximately 10:55:40 G.C.T., 1951 April 10. In $a$ the traces are, respectively: N-S electromagnetic strain seismograph, vertical-component electromagnetic pendulum, E-W electromagnetic pendulum, N-S electromag-

1950 MAY 17

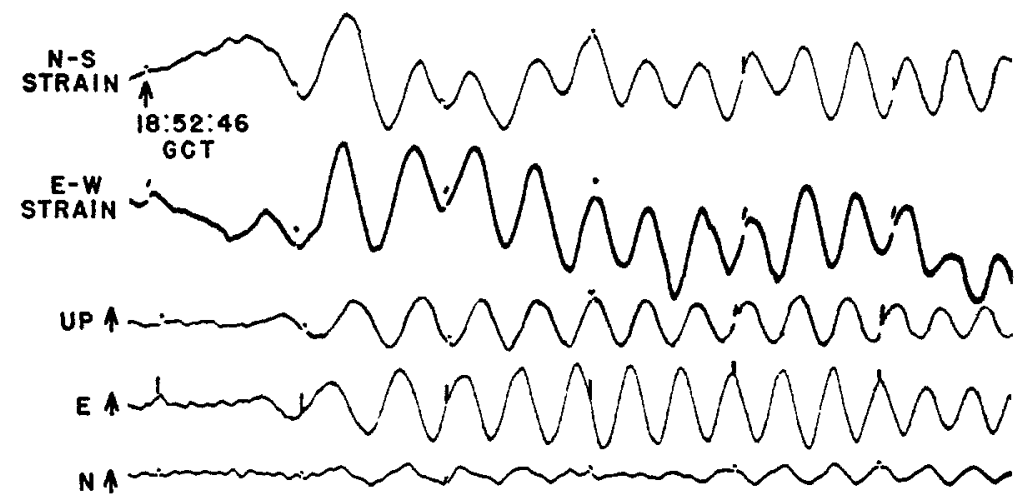

Fig. 3. Pasadena seismograms of the same earthquake as in figure 2. Reading from top to bottom, the traces are: $1, \mathrm{~N}-\mathrm{S}$ strain seismograph; $2, \mathrm{E}-\mathrm{W}$ strain seismograph; 3, vertical pendulum; 4, E-W pendulum; $5, \mathrm{~N}-\mathrm{S}$ pendulum; Benioff seismographs. $T_{g}=$ approximately 90 seconds.

netic pendulum. All four instruments record with galvanometers having approximately 90 seconds period. Figure $4, b$ shows two traces of the same earthquake recorded by an electromagnetic vertical-pendulum seismograph and an electromagnetic N-S strain seismograph, both having galvanometers of 18 seconds period. The trace phases of the N-S electromagnetic strain and the electromagnetic verticalcomponent pendulum seismographs are identical, and consequently it may be concluded that the wave train represents a Rayleigh wave group. The trace phases of the two horizontal-component pendulum instruments (fig. 4, a) agree with each other but are displaced $90^{\circ}$ ahead of the strain and vertical traces, indicating that these Rayleigh waves arrived from the southwest direction.

Figure 4, $c$ represents selected portions of seismograms of the earthquake of 1951 April 14, $13^{\mathrm{h}} 33^{\mathrm{m}} .0$, which occurred in East Siberia about $\phi=61^{\circ}, \lambda=136^{\circ} \mathrm{E}$, $\Delta=68^{\circ}$, and azimuth approximately $330^{\circ}\left(\mathrm{N} 30^{\circ} \mathrm{W}\right)$. The upper traces were recorded with a galvanometer of 18 seconds period on a vertical-component electromagnetic seismograph, and the lower traces were recorded with an electromagnetic strain seismograph and similar galvanometer. At approximately 14:02 in the first line, long-period Love waves are shown with no evidence of vertical movement. The horizontal-component pendulum seismograms (not reproduced here) confirm 
this conclusion. The remainders of the seismograms are composed principally of Rayleigh waves. Although the wave forms of the traces of the two instruments are not identical, the phases of the two agree.

Figure 5 shows a comparison between seismograms of the five instruments, discussed earlier, for the earthquake of 1950 May 26, 1:17:25 \pm G.C.T., which occurred near the Loyalty Islands at approximately $\phi=21^{\circ} \mathrm{S}, \lambda=169^{\circ} \mathrm{E}, \Delta=88^{\circ}$ and
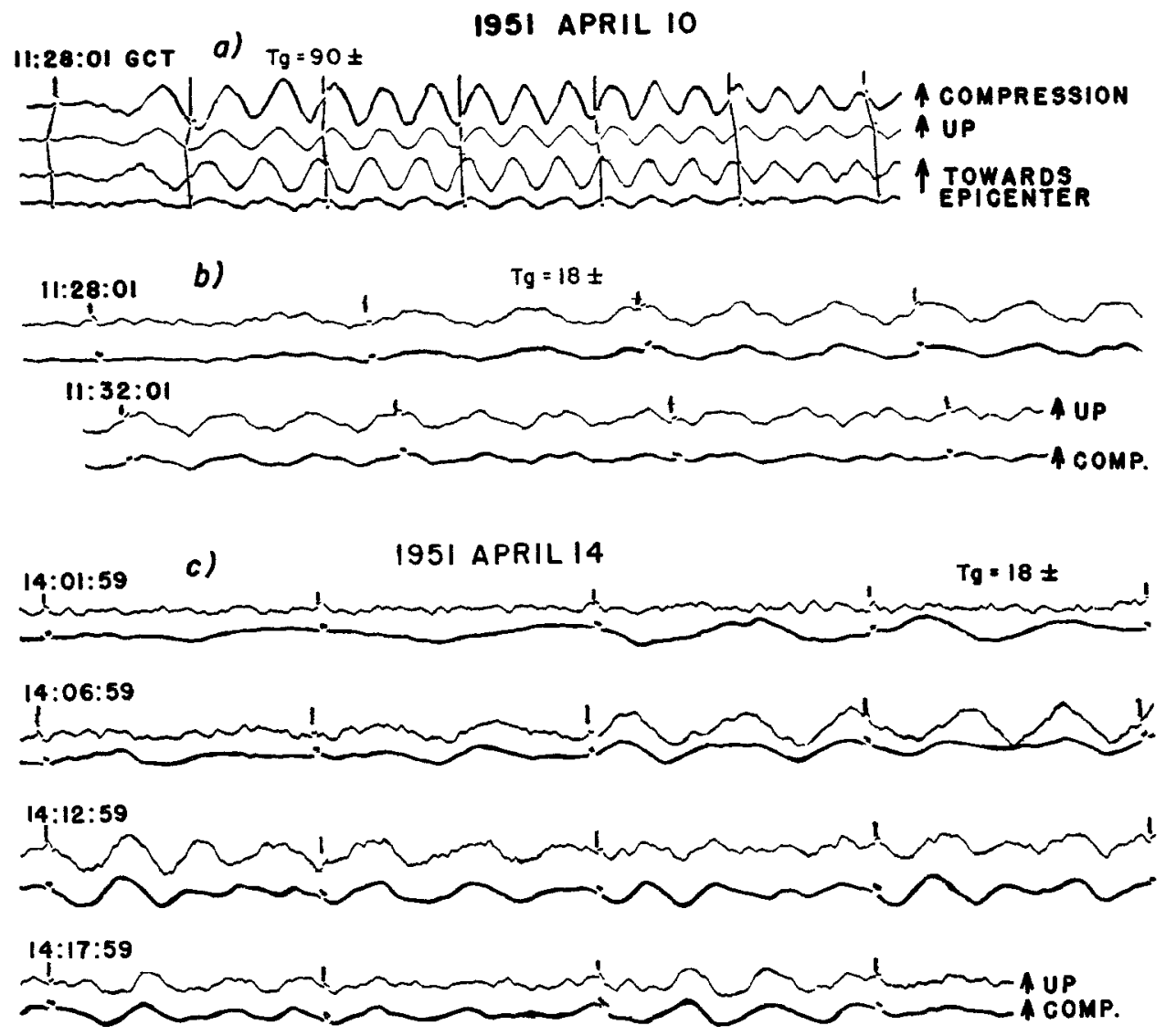

Fig. 4. Pasadena seismograms of surface waves.

azimuth $=240^{\circ}\left(\mathrm{W} 30^{\circ} \mathrm{S}\right)$. The long-period Love $(\mathrm{G})$ waves begin at approximately $1^{\mathrm{h}} 54^{\mathrm{m}}$ and the Rayleigh waves at approximately $1^{\mathrm{h}} 57^{\mathrm{m}}$. It can be seen that the strain seismograph traces of the first large wave (G) are in phase opposition which indicates a shear wave, while in the later group (continuing for more than one hour) the traces are in phase and so indicate Rayleigh waves. The shorter-period wave in the vertical component trace is probably some late body wave, since in the Rayleigh wave group the strain traces are of like phase and correspond with the vertical pendulum. The $\mathrm{E}-\mathrm{W}$ and $\mathrm{N}-\mathrm{S}$ pendulum traces have opposite phases in the $\mathrm{G}$ wave. In the Rayleigh wave group the two horizontal pendulum traces have like phases which precede the trace phases of the vertical pendulum and of the strain instrument. 
A last example is given in figure 6, which represents seismograms from the same group of instruments as in preceding discussion. This shock occurred in the East Indies, 1950 October $8,3^{\mathrm{h}} 23^{\mathrm{m}} 9 \pm^{\mathrm{s}}, \phi=4^{\circ} \mathrm{S}, \lambda=120^{\circ} \mathrm{E}, \Delta=111^{\circ}$, azimuth approximately $278^{\circ}\left(\mathrm{W} 8^{\circ} \mathrm{N}\right)$. The seismograms show Rayleigh waves beginning at approximately $4^{\mathrm{h}} 14^{\mathrm{m}}$. For these waves the traces from all instruments are in accorddance with predictions. At approximately 4:09, the time at which $\mathrm{G}$ should arrive, there appears on the $\mathrm{N}-\mathrm{S}$ strain trace and on the $\mathrm{N}-\mathrm{S}$ pendulum trace a wave of

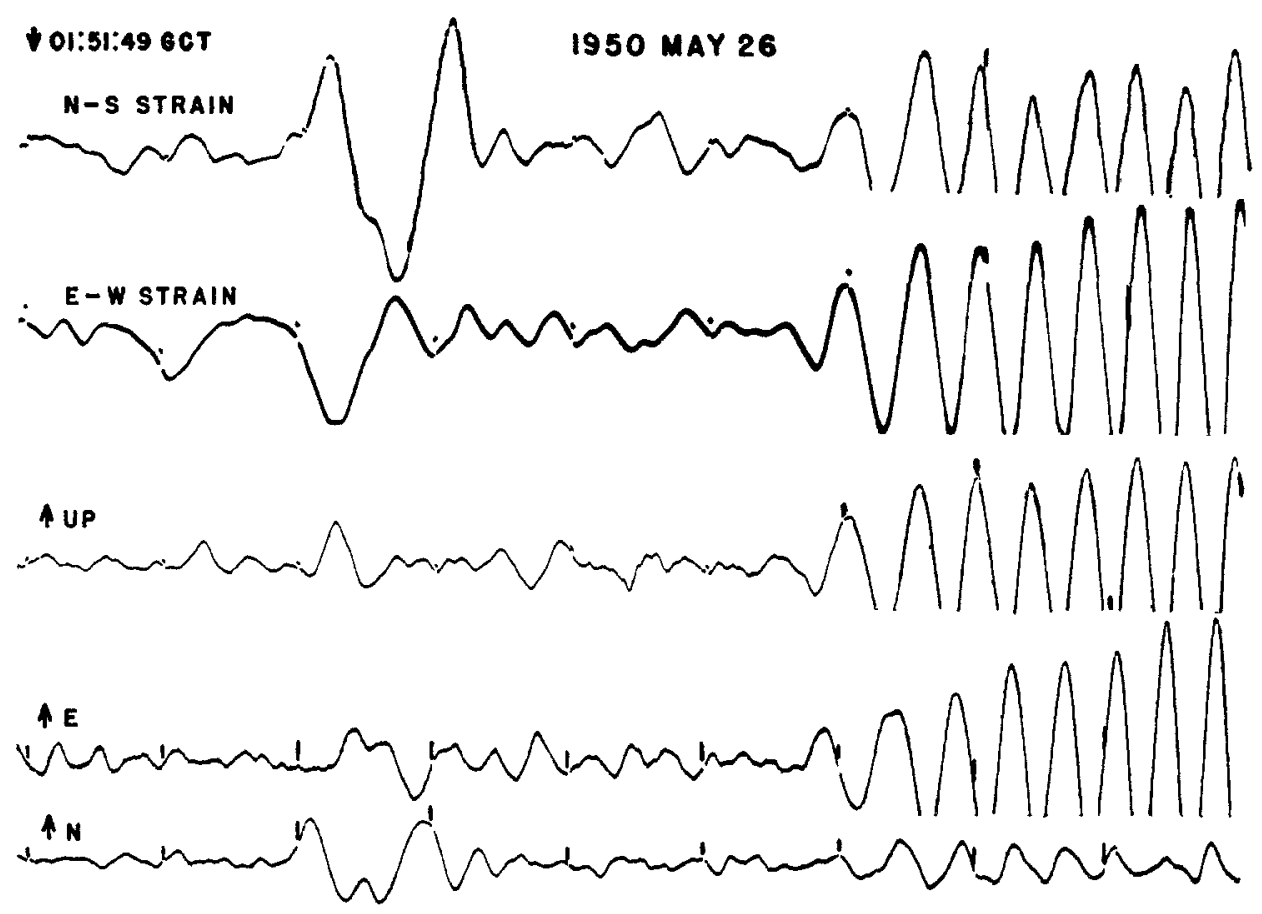

Fig. 5. Pasadena seismograms of surface waves. Trace sequence as in figure 3. $T_{o}=$ approximately 90 seconds.

approximately 90 seconds period. It is not recorded on the vertical pendulum trace and is poorly recorded, if at all, on the $\mathrm{E}-\mathrm{W}$ pendulum trace. If this wave is a $\mathrm{G}$ (or Love wave) the pendulum response is normal. On the other hand, in this case the $\mathrm{E}-\mathrm{W}$ strain should be of equal amplitude (except for a magnification factor) and of opposite phase from that of the N-S strain. However, on the E-W strain seismograph trace this wave is entirely absent although the long-period Rayleigh waves are well recorded. Clearly this represents an anomaly of some sort. It may result from the fact that the waves, obliquely incident at the Pacific Coast, impinge on a surface of discontinuity. In any case, further study of $\mathrm{G}$ waves recorded on these instruments is required.

\section{Summary}

The investigations reported in this paper clearly exhibit advantages gained from the use of strain seismographs and of vectorial recorders. The relationship between the response of strain and pendulum seismographs is peculiarly well adapted to demonstrate the characteristic elliptical motion of the ground particles in Rayleigh waves. 
1950 OCT. 8

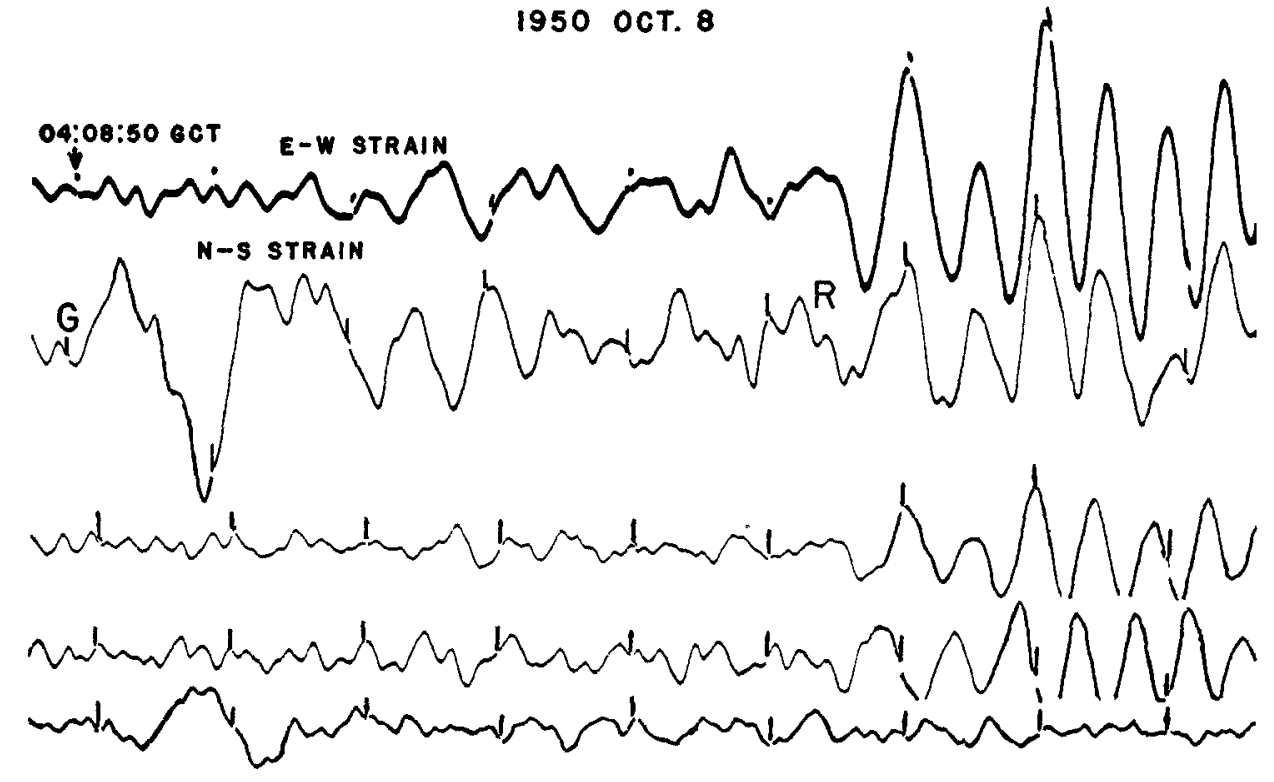

Fig. 6. Pasadena seismograms of surface waves. Sequence of last three traces same as in figure 3.

Further and more detailed investigations concerning the effects of crustal inhomogeneities on surface waves are needed. It should be possible to use the vectorial recorder either with vertical- and horizontal-pendulum seismometers or with verticalpendulum and horizontal-strain seismometers to determine the actual shape of the Rayleigh wave ellipses as well as the inclination of their major axes. Some of our preliminary observations suggest that the major axis may be inclined slightly to the vertical.

California Institute of Technology

Pasadena, CaLifornia

(Division of Geological Sciences, contribution no. 582) 